\title{
Cinética de absorción de plomo en especies vegetativas previo a procesos de fitorremediación de suelos altamente contaminados
}

\author{
Lead absorption kinetics in vegetative species prior to phytoremediation \\ processes in highly contaminated soils

\section{Cinética de absorção de chumbo em espécies vegetativas antes de processos de fitorremediação em solos altamente contaminados}

\author{
Elena Coyago \\ ecoyagoc@ups.edu.ec
}

Sara Bonilla

elena.coyago@hotmail.com

\author{
Universidad Politécnica Salesiana, Sede Quito, Ecuador
}

Artículo recibido septiembre 2018, arbitrado octubre 2018 y publicado en enero 2019

\begin{abstract}
RESUMEN
La industrialización, el incremento de la población y la inapropiada disposición de residuos peligrosos han generado episodios de contaminación severos en suelo, aire y agua. El presente estudio tuvo como objetivo evaluar la cinética de absorción de metales pesados en tres especies vegetativas Amaranto Hybridus (amaranto), Beta Vulgaris (acelga) y Medicago sativa (alfalfa) trasplantadas en suelos altamente contaminados a concentraciones $2,5,5$ y $10 \%$ de plomo y evaluadas bajo la técnica de espectrofotometría de absorción atómica a la llama previo proceso de digestión ácida; la evolución del proceso de absorción se cuantificó a los $0,20,30,45,60$ y 90 días. Los resultados encontrados reportaron que a mayor valor de biomasa mayor absorción de plomo, esto se debió a que el plomo contó con mayor espacio para su deposición, por otra todas las especies presentaron etapas de desintoxicación cuando llegaron a un máximo de absorción siendo menos pronunciada esta etapa en la alfalfa y la acelga. En el caso de especies con mayor biomasa la etapa de desintoxicación fue de aproximadamente 10 días con porcentajes de fitorremediación del 90\%, mientras que las especies con menor biomasa luego de los 70 días no lograron recuperar su poder de absorción reportando valores máximos de fitorremediación del $25 \%$, por tanto todo proceso de fitorremediación debería ir acompañado de la cinética de absorción del metal pesado en las especies vegetativas ya que las etapas de desintoxicación hacen menos efectivo el proceso de remediación de suelos, existiendo la probabilidad de llegar a índices muy bajos de descontaminación.
\end{abstract}

Palabras clave: Fitorremediación; contaminación del suelo; cinética de absorción

\begin{abstract}
Industrialization, the increase in population and the inappropriate disposal of hazardous waste have generated severe episodes of contamination in soil, air and water. The present study aimed to evaluate the absorption kinetics of heavy metals in three vegetative species Amaranth Hybridus (amaranth), Beta Vulgaris (chard) and Medicago sativa (alfalfa) transplanted in highly contaminated soils at concentrations 2.5, 5 and $10 \%$ lead and evaluated under the flame atomic absorption spectrophotometry technique after acid digestion process; the evolution of the absorption process was quantified at $0,20,30,45,60$ and 90 days. The results found reported that the higher the biomass value, the greater the absorption of lead, this was due to the fact that the lead had more space for its deposition, on the other hand, all the species presented detoxification stages when they reached a maximum absorption, being less pronounced this stage in alfalfa and chard. In the case of species with higher biomass, the detoxification stage was approximately 10 days with phytoremediation percentages of $90 \%$, while species with lower biomass after 70 days failed to recover their absorption power, reporting maximum values of phytoremediation of $25 \%$, therefore, all phytoremediation process should be accompanied by the kinetics of heavy metal absorption in vegetative species since the detoxification stages make the soil remediation process less effective, with the probability of reaching very low levels of decontamination.
\end{abstract}

Key words: Phytoremediation; soil contamination; absorption kinetics 


\section{RESUMO}

A industrialização, o aumento da população e o descarte inadequado de resíduos perigosos geraram episódios graves de contaminação no solo, no ar e na água. 0 presente estudo teve como objetivo avaliar a cinética de absorção de metais pesados em três espécies vegetativas Amaranth Hybridus (amaranto), Beta Vulgaris (acelga) e Medicago sativa (alfafa) transplantadas em solos altamente contaminados nas concentrações $2,5,5$ e $10 \%$ chumbo e avaliado pela técnica de espectrofotometria de absorção atômica com chama após processo de digestão ácida; a evolução do processo de absorção foi quantificada aos $0,20,30,45$, 60 e 90 dias. Os resultados encontrados relataram que quanto maior o valor da biomassa, maior a absorção de chumbo, isso se deve ao fato de o chumbo ter mais espaço para sua deposição, por outro lado, todas as espécies apresentaram estágios de desintoxicação quando atingiram uma absorção máxima, sendo menos pronunciado. estágio em alfafa e acelga. No caso de espécies com maior biomassa, o estágio de desintoxicação foi de aproximadamente 10 dias com porcentagens de fitorremediação de $90 \%$, enquanto espécies com menor biomassa após 70 dias falharam em recuperar seu poder de absorção, relatando valores máximos de fitorremediação de $25 \%$, portanto, todo processo de fitorremediação deve ser acompanhado pela cinética da absorção de metais pesados nas espécies vegetativas, uma vez que as etapas de desintoxicação tornam o processo de remediação do solo menos eficaz, com probabilidade de atingir níveis muito baixos de descontaminação.

Palavras-Chave: Fitorremediação; contaminação do solo; cinética de absorção

\section{INTRODUCCIÓN}

La EPA define a la biorremediación como manipulación de sistemas biológicos para efectuar cambios en el ambiente (Cruz y Guzmán, 2007), dependiendo del medio que se utilice se clasifica en Fitorremediación uso de plantas verdes (Puga, Sosa, Lebgue, Quintana, y Campos, 2006; Agudelo, Macias, y Suárez, 2009). Además, se tiene a la Biorremediación uso de animales, y también la Biorremediación microbiana que habla acerca del uso de bacterias acumuladoras (Datta, Geiger, y Sarkar, 2013)

Especies herbáceas, arbustivas y árboles pueden utilizarse para la fitorremediación (Peng, Yang, y Deng, 2012), existiendo varios factores que influyen en el proceso de absorción como tipo de planta, biomasa disponible, entre otros factores (Nouri, y otros, 2011; Volke, Velasco, y Pérez, 2005). Una de las limitaciones de la fitorremediación incluye procesos de recuperación de suelos con poca profundidad, por ello es muy usual el uso de especies herbáceas (Lee, 2013).

La remoción de contaminantes dentro del metabolismo de las plantas se realiza por procesos de fitoextracción, fitoestabilización, fitoinmovilización, fitovolatilización, fitodegradación, fizofiltración y fitoestimulación (Diez, Kidd, y Monterroso, 2009) (Babula, y otros 2012) y estos procesos son acompañados por los mecanismos de absorción, excreción y desintoxicación de contaminantes en la planta (Lee, 2013), existiendo plantas que pueden acumular gran cantidad de metales pesados las cuales se denominan hiperacumuladoras y hoy en día constituyen temas de estudio (Sarvajeet, y otros, 2012), además del uso de potenciadores de absorción de metales pesados o agentes quelantes como el EDTA (ácido etilendiaminotetraacético) que es un producto químico de baja biodegradabilidad y se utiliza como quelante del plomo, convirtiendose en una contaminación secundaria para los suelos a recuperar (Seth, Misra, y Singh, 2011; Manasi, Rajesh, y Rajesh, 2014).

Este estudio tiene como propósito evaluar la cinética de absorción de metales pesados en tres especies vegetativas Amaranto Hybridus (amaranto), Beta Vulgaris (acelga) y Medicago sativa (alfalfa) a fin de determinar los niveles de contaminación del plomo y su impacto demandan nuevas alternativas para la reducción de la contaminación que inclusive en la actualidad son limitadas (Manasi, Rajesh, y Rajesh, 2014), por lo cual es importante que estas alternativas mantengan las condiciones físicas-biológicas del suelo y la biorremediación es una alternativa viable, 
de menor costo (Adesodun, Atayese, Abbaje, Osadiaye, Mafe, y Soretire, 2010) y que puede llevase a cabo en el mismo lugar utilizando plantas nativas que soporten el estrés ambiental del lugar (Gupta, Huang, y Corpas, 2013).

\section{MATERIALES Y MÉTODOS}

Para la selección de especies utilizadas para la fitoremediación en suelos contaminados previo a la experimentación, se realizó una investigación bibliográfica y se seleccionó especies que han sido utilizadas en los procesos de fitorremediación considerando los siguientes aspectos:

- Resistentes a condiciones ambientales

- Resistentes a plagas

- Cultivos de ciclo corto

Las plántulas utilizadas para el proceso de fitorremediación correspondieron a semillas certificadas adquiridas en el Instituto Nacional Autónomo de Investigaciones Agropecuarias (INIAP) estación Santa Catalina-Quito, Ecuador.

\section{Construcción de semilleros}

En la construcción de semilleros la germinación de cada una de las especies seleccionadas, se estableció dos tipos de sustrato: a). Tierra negra sin tratamiento; y b). Mezcla de tierra negra, cascajo y abono orgánico en proporciones 3:1:1 (tierramezcla). A cada sustrato se cuantificó el pH utilizando la norma AST D4972 (RECNAT, 2002; ASTM, 2014). El proceso de germinación se realizó bajo las mismas condiciones de temperatura y humedad dejando transcurrir el tiempo necesario hasta que las plántulas alcancen la madurez para el trasplante, aproximadamente cuatro semanas.
Exposición de plantas seleccionadas a suelos con diferentes concentraciones de plomo

Según estudios realizados en las inmediaciones de industrias que utilizaron plomo en sus procesos determinaron concentraciones de plomo alrededor de $51000 \mathrm{mg} \mathrm{Pb} / \mathrm{kg}$ de suelo en Canadá y $37600 \mathrm{mg} \mathrm{Pb} / \mathrm{kg}$ de suelo en el Salvador según lo expuesto por (Herrera, 2009), por tanto se seleccionó concentraciones de 2,5; 5 y $10 \%(\mathrm{p} / \mathrm{p})$. Las plantas expuestas al plomo fueron monitoreadas a $\operatorname{los} 0,20,30$, 45, 60 y 90 días.

Cuantificación de plomo, utilizando la técnica de espectrofotometría de absorción atómica a la llama

El monitoreo de absorción de plomo se realizó empleando el método EPA 3010a, rev. 01, 1992; Standard Methods ed-212005, 3500 (EPA, 2005), el cual señala una digestión ácida y la posterior cuantificación en un espectrofotómetro de absorción atómica. La cuantificación se realizó por triplicado y con selección aleatoria de las muestras a analizar. Los ítems cuantificados fueron los siguientes:

- Suelo sin contaminante

- Suelos contaminados a una concentración de 2,5; 5 y 10\% (p/p) de plomo.

- Suelos contaminados luego del proceso de fitorremediación

- Plántulas a ser trasplantadas sin exposición de plomo.

- Plantas expuestas a las diferentes concentraciones de plomo y monitoreadas a 20, 30, 45, 60 y 90 días.

El proceso de digestión se realizó por 35 min en un digestor marca Buchi Scrubber Modelo B414 previa adición de 50 mL de ácido nítrico Merck de pureza 69\%, seguido de un proceso de filtrado, aforo a 
$50 \mathrm{~mL}$ con ácido nítrico al 3\% y la posterior lectura en el espectrofotómetro de absorción atómica.

\section{Fitorremediación}

La fitorremediación de suelos se determinó cuantificando las concentraciones de contaminante presente en cada uno de los suelos seleccionados utilizando la técnica de absorción atómica y empleando el método EPA descrito en el numeral 2.4, para posteriormente calcular el porcentaje de fitorremediación como se muestra en la ecuación siguiente.

$$
\% F=100-\frac{C o-C f}{C o} * 100
$$

Donde:

$$
\text { \% F = \% Fitorremediación }
$$

Co $=$ Concentración inicial de contaminante en el suelo $\mathrm{Cf}=$ Concentración final de contaminante en el suelo

La concentración final obtenida se sometió a comparación con el valor de 100 $\mathrm{mg} / \mathrm{Kg}$ de plomo establecido en la Tabla 3 , Anexo 2, libro 6 del TULSMA y se calculó el número de cultivos aproximados para lograr cumplir la normativa.

\section{RESULTADOS Y DISCUSIÓN}

\section{Selección de especies utilizadas para la fitorremediación en suelos contaminados}

Estudios realizados por autores como: (López y Espinosa, 2006; Suthar, Memon, y Hassa, 2014; López y Torija, 2006; Mateo, 2008; Salas y Boradonenko, 2009; Peralta, 2012; Chinmayee, y otros, 2012; Flores, 2010), entre otros, señalan que existen más de 1500 especies vegetativas utilizadas en los procesos de fitorremediación siendo las más comunes las especies desarrolladas en los lugares en que se realizará el proceso de recuperación del suelo. Dentro de las especies más usadas en los procesos de fitorremediación se encuentra el amaranto
(Amaranthus hybridus) y el girasol (Helianthus anuas); estos cultivos se caracterizan por ser bianuales y presentan resistencia a la sequía, además son cultivadas en zonas tropicales y templadas (Salas y Boradonenko, 2009; Peralta, 2009; Peralta, 2012; Chinmayee, y otros, 2012). Por otra parte la alfalfa y acelga se caracterizan por ser especies que absorben metales pesados según expone la Agencia Española de Seguridad Alimentaria y Nutrición (AESAN, 2012) y son muy utilizados a nivel mundial como forraje para animales y alimento para humanos.

Por tanto, por tratarse de especies de ciclo corto, resistentes a la sequía, adaptables a diferentes climas y que frecuentemente son cultivadas en todo el territorio ecuatoriano, se seleccionó al amaranto, la acelga y la alfalfa para someterlas al proceso de fitorremediación.

\section{Construcción de semilleros}

Los semilleros fueron portátiles, construidos para alojar los dos tipos de sustrato establecido y para cada una de las especies seleccionadas como se muestra en la Figura 1. Cada semillero presentó 
orificios para drenaje de agua en exceso y se cuantificó el pH dando como resultados valores de 7,1 para tierra negra y 7,5 para tierra mezcla, estos valores sugieren que se trata de un suelo neutro y medianamente alcalino respectivamente según establece la norma ASTM D4972.

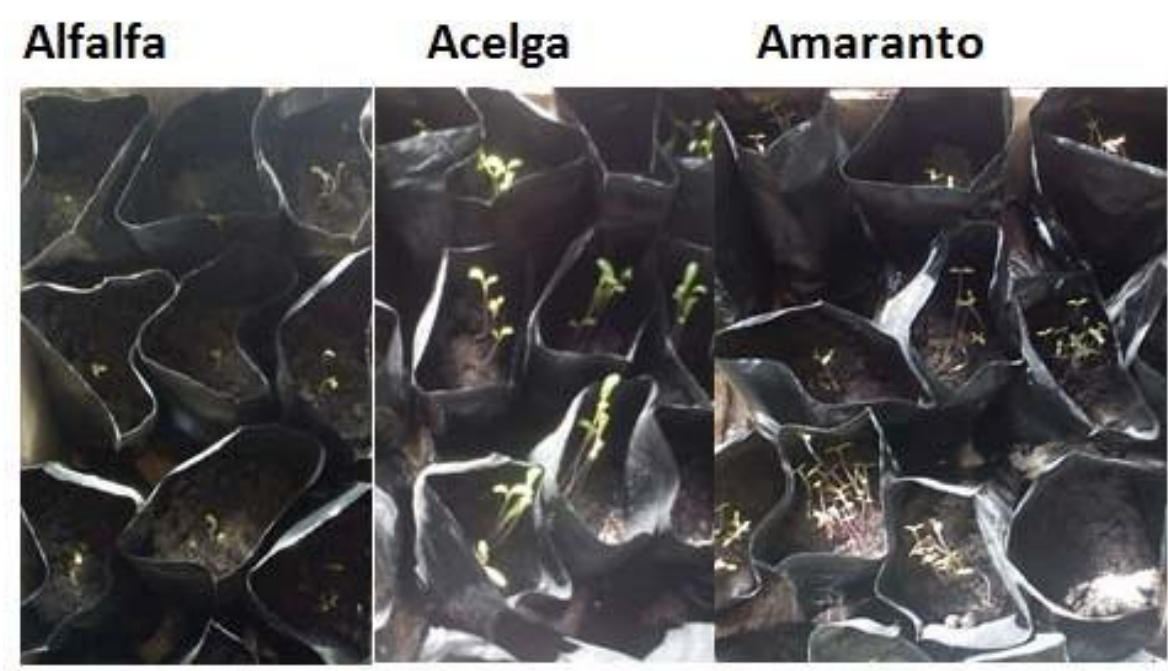

Figura 1. Semilleros.

Luego de haber transcurrido cuatro semanas el follaje, grosor de las plántulas, sistema radicular y altura de las plantas germinadas en tierra-mezcla fue mayor que las plántulas germinadas y desarrolladas en tierra negra como se observa en la Figura 2, por tanto la cantidad de biomasa generada se ve influencia por la etapa de desarrollo inicial que tiene la planta y por consiguiente la adición de abono en esta etapa es muy importante como señalan (Guanopatín, 2012; Chamizo, y otros 2009; Redín, 2009).

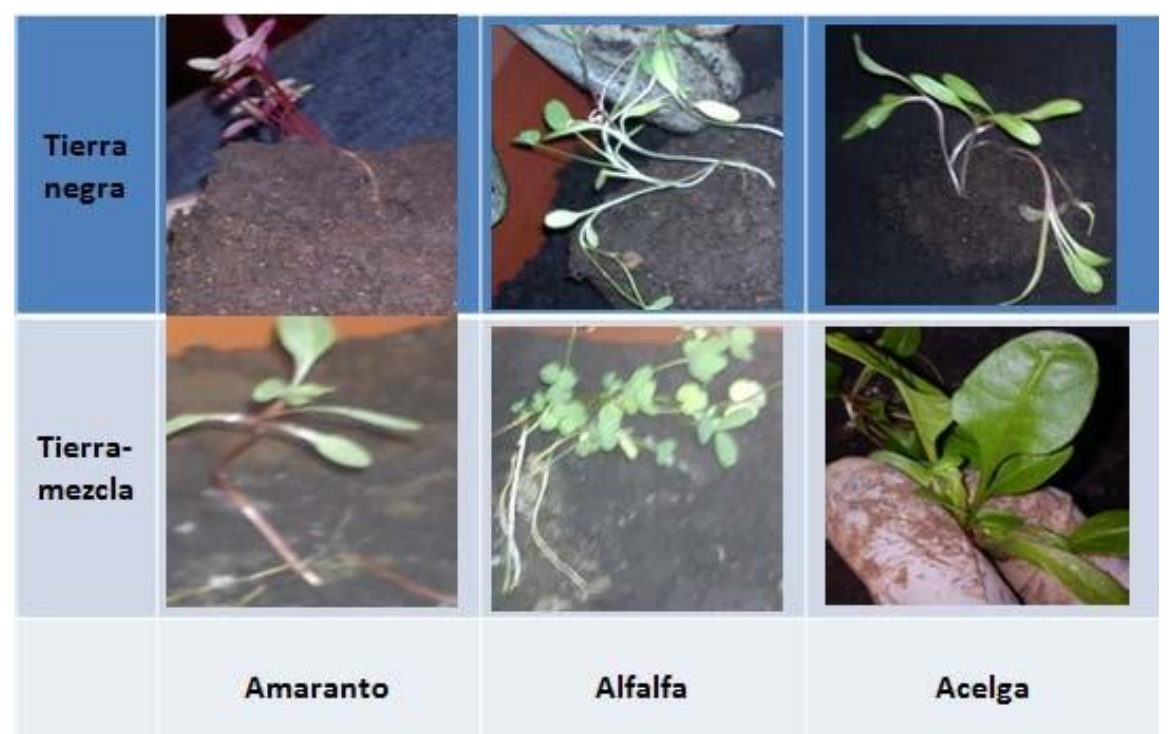

Figura 2. Plántulas listas para el trasplante. 
Exposición de plantas seleccionadas a suelos con diferentes

\section{concentraciones de plomo}

El sustrato contaminado con plomo en concentraciones de 2,5, 5 y $10 \%(\mathrm{p} / \mathrm{p})$, fue preparado utilizando cenizas de plomo recicladas de baterías automotrices y tierra cangagua que es un suelo común en el norte y sur de Quito según lo expuesto por (Jácome, 2011) como se observa en la Tabla 1. A cada uno de los suelos contaminados se determinó el valor de $\mathrm{pH}$ reportando que se trató de suelos moderadamente ácidos según lo expuesto por la norma (ASTM, 2014; NOM, 2002). En los sustratos contaminados se replantó las especies que fueron germinadas $\mathrm{y}$ desarrolladas por cuatro semanas; cada especie sometida al proceso de contaminación resistió al cambio presentando una tasa de mortalidad nula.

Tabla 1. Valores de $\mathrm{pH}$ en suelos contaminados

\begin{tabular}{rc}
\hline \multicolumn{1}{c}{ Ítem } & pH \\
\hline Suelo 2,5\% Pb (M1) & 6,2 \\
Suelo 5\% Pb (M2) & 6,0 \\
Suelo 10\% Pb (M3) & 6,0 \\
\hline
\end{tabular}

Cuantificación de plomo, utilizando la técnica de espectrofotometría de absorción atómica a la llama

La cuantificación de plomo de las diferentes especies sembradas en tierra negra como se observa en la Figura 3, reportaron patrones de absorción continuo para concentraciones bajas, mientras que para concentraciones medias el amaranto $\mathrm{y}$ la acelga presentaron una etapa de desintoxicación a los 30 días, este mismo comportamiento se observó en concentraciones altas, por panto a pesar de la escasa biomasa generada las especies vegetativas absorbieron concentraciones importantes de contaminante hasta saturar su estructura, tiempo en el cual se generó la etapa de desintoxicación. 

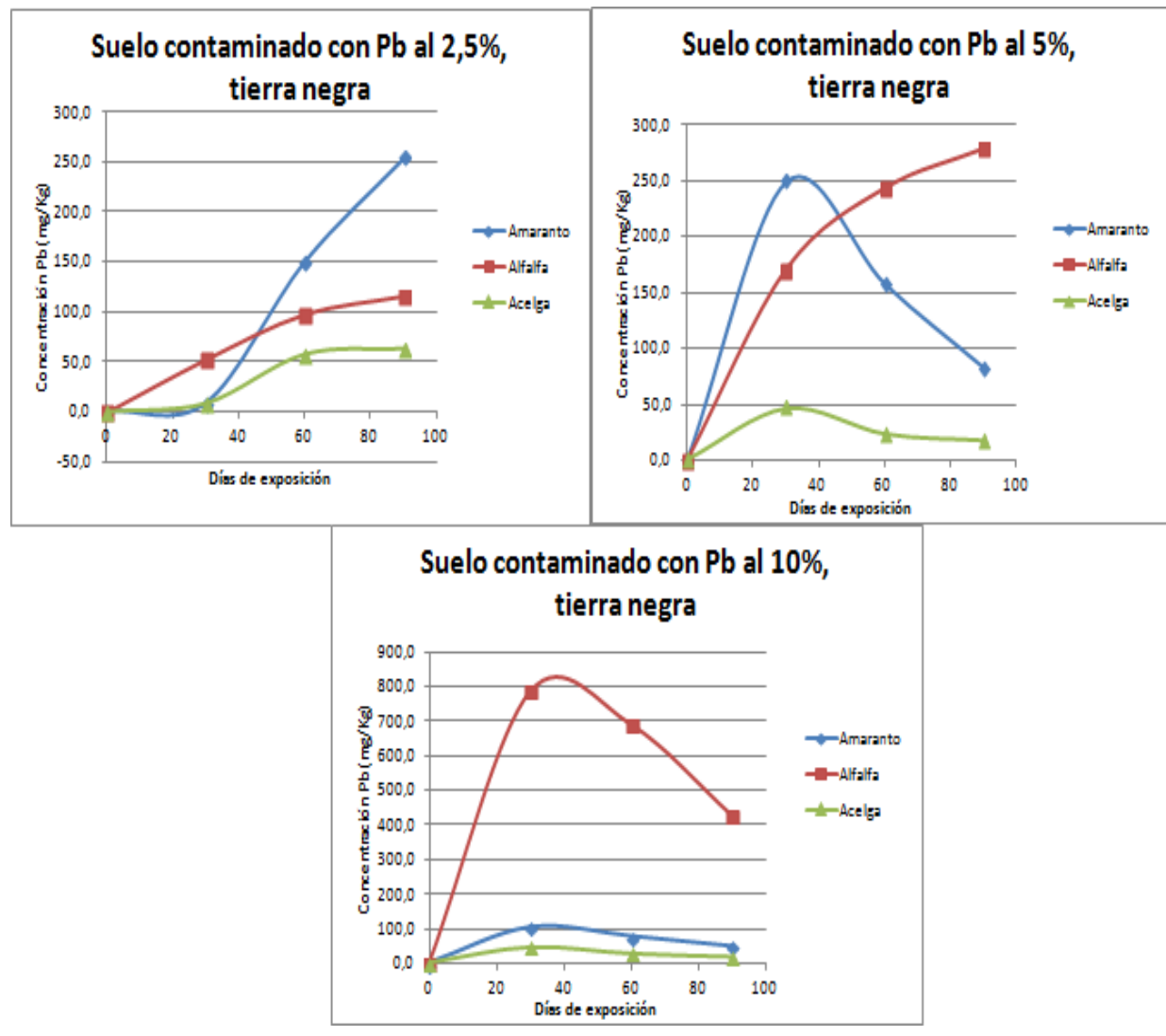

Figura 3. Plantas germinadas en tierra negra a diferentes concentraciones de contaminación en función de los días de exposición.

En plantas germinadas en tierra mezcla se observó que el vigor de las plantas fue mayor que las plantas germinadas en tierra negra y la absorción de contaminante fue mayor. A los 20 días como se observa en la Figura 4 se presentaron etapas de desintoxicación para todas las especies el cual continuó hasta los 45 días, dicha cinética de absorción de plomo cumplió lo expuesto por (Pacheco, Pimentel, y Roque, 2010) en el estudio "Cinética de la bioadsorción de iones cadmio (II) y plomo (II) de soluciones acuosas por biomasa residual de café (Coffea arabica L.) en la que señala que la cinética de absorción de plomo se ajusta en mejor medida a un modelo pseudo primer orden en donde existe una etapa inicial de absorción continua del contaminante y una posterior recesión del proceso.
A los 60 días de exposición las plantas de alfalfa mostraron una gran capacidad para absorber plomo, el amaranto menor capacidad y la acelga capacidad media, quedando evidenciado que el proceso de fitorremediación no solo depende del tipo de planta utilizada, sino también del tiempo que el proceso se demore, ya que mientras más tiempo esté en contacto el metal pesado con el suelo puede existir una depuración del mismo o una recontaminación como señala (Singh y Fulekar, 2012), por tanto los procesos de fitorremediación sugieren la necesidad de conocer previamente la cinética de absorción del metal pesado para someter a una especie vegetativa al proceso. 
La alfalfa y la acelga germinadas en tierra-mezcla presentaron facilidad de absorción, esto permite evidenciar que son especies que facilitan el proceso y existe la necesidad de determinar que al llegar a la etapa de maduración se podría desprender los tallos generados para que retoñen nuevas hojas y que el proceso de descontaminación continúe sin tener que replantar nuevas especies.

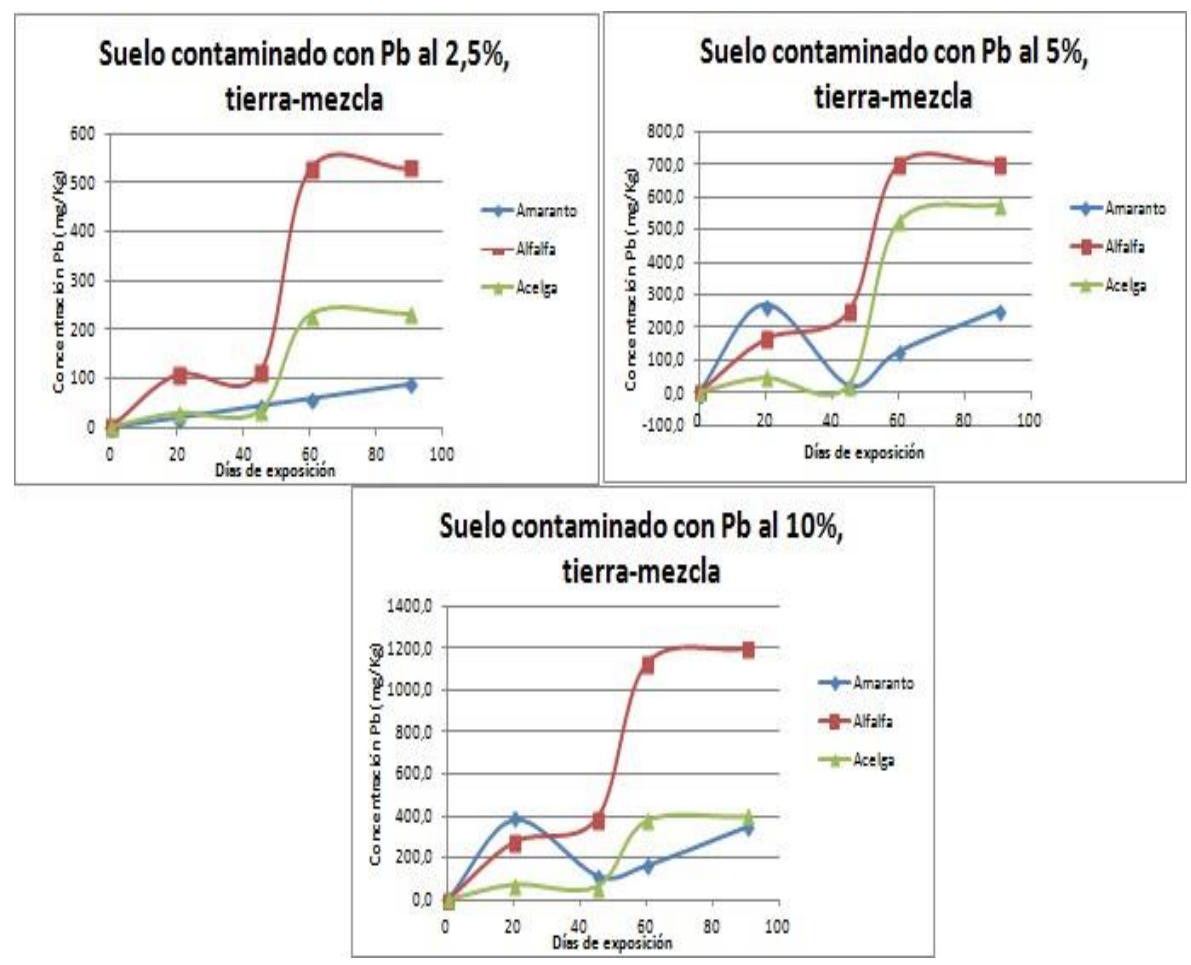

Figura 4. Plantas germinadas en tierra mezcla a diferentes concentraciones de contaminación en función de los días de exposición.

\section{Fitorremediación}

La fitorremediación de suelos utilizando plantas germinadas en tierra negra presentó valores inferiores al $25 \%$ de remoción de contaminante como se observa en la Figura 5, este fenómeno puede deberse a que las plantas no desarrollaron importante biomasa durante la germinación y en el tiempo de exposición al contaminante como se observa en la Figura 2 , además las etapas de desintoxicación pueden recontaminar el suelo provocando que los índices de remoción de plomo sean bajos, mientras que las plantas germinadas en tierra-mezcla presentaron valores de fitorremediación superiores al $37 \%$ llegando a porcentajes de remoción del $99 \%$ en el caso de la alfalfa, este proceso pudo deberse a que la planta que no presenta abundante biomasa genera procesos de intoxicación más rápido que las especies que presentan mayor biomasa según lo expuesto por (DeSouza, y otros, 2012). 


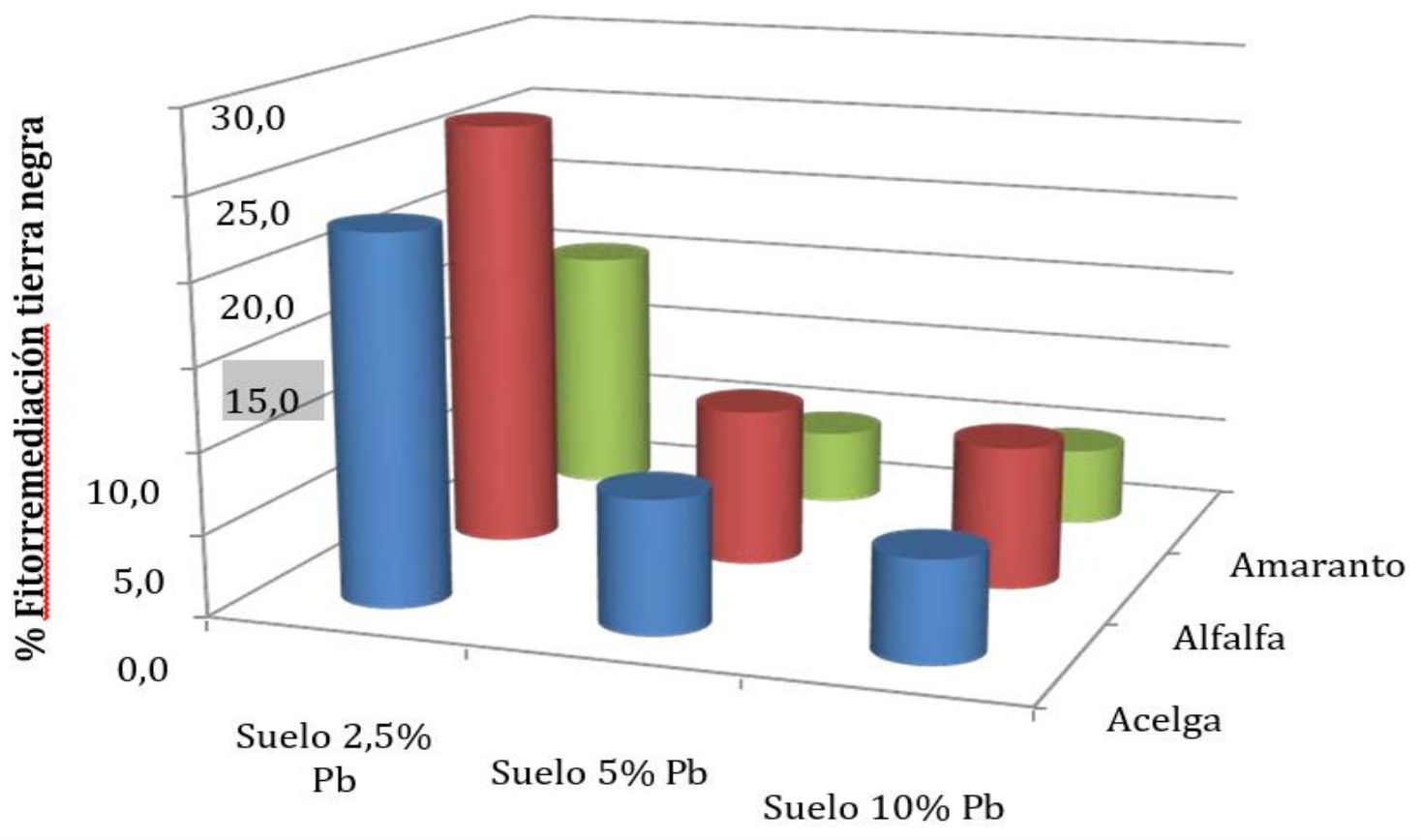

Figura 5. Porcentaje de Fitorremediación con plantas germinadas en tierra negra.

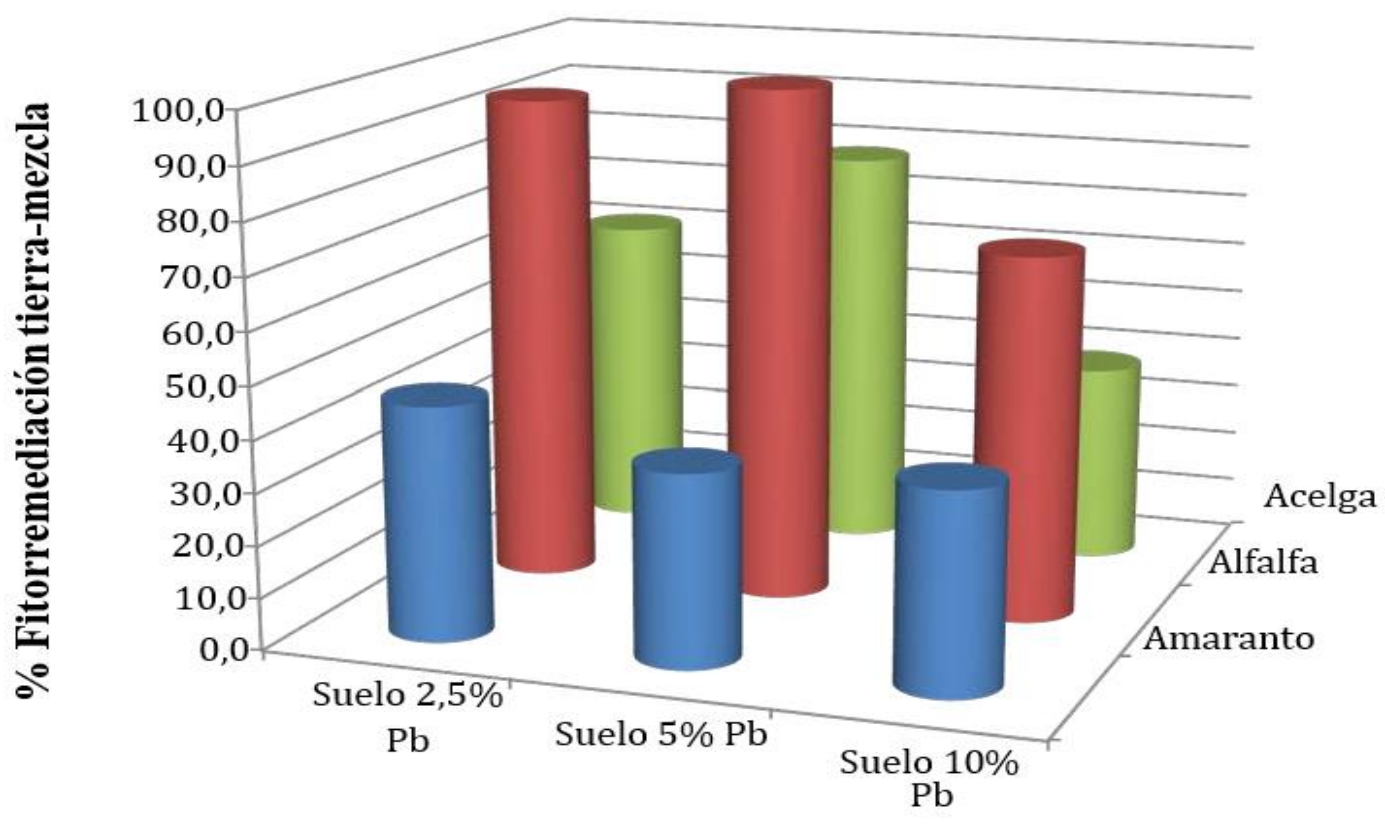

Figura 6. Porcentaje de fitorremediación con plantas germinadas en tierra-mezcla. 


\section{CONCLUSIONES}

Los procesos de fitorremediación están ligados a la cinética de absorción de cada especie y a los procesos de desintoxicación de las mismas. Así las especies Amaranthus hybridus (amaranto) y Beta vulgaris var. cicla (acelga) presentan un proceso de desintoxicación depositando el contaminante nuevamente en el suelo.

Medicago sativa (alfalfa) y Beta vulgaris var. cicla (acelga) presentaron etapas de absorción continua durante el tiempo de estudio (90 días) presentando un proceso de absorción de plomo sin mostrar desintoxicación de la planta, esto sugiere que puede tratarse de una especie hiperacumuladora.

La adición de abono o nutrientes previo a la germinación de las especies, se convierte en un factor determinante para la fitorremediación, ya que esto ayuda a que las plantas presenten abundante sistema radicular, un mayor crecimiento, frondosidad de la planta y mejores características al momento del trasplante.

El proceso de fitorremediación es una técnica relativamente nueva en el país y económicamente rentable, especialmente porque se puede trabajar con especies menores de ciclo corto

\section{REFERENCIAS}

Adesodun, J., Atayese, M., Abbaje, T., Osadiaye, B., Mafe, O., y Soretire, A. (2010). Phytoremediation Potentials of Sunflowers (Tithonia diversifolia and Helianthus annuus) for Metals in Soils Contaminated with Zinc and Lead Nitrates. Water Air Sail Pollut, 7

AESAN. (2012). Alimentos peligrosos. Agencia Española de Seguridad Alimentaria y Nutrición, 10

Agudelo, L., Macias, K., y Suárez, A. (2009). Fitorremediación: la alternativa para absorber metales pesados de los biosólidos. Red Revista Lasallista, 6 .
ASTM. (2014). ASTM D4972 Standard test method for $\mathrm{pH}$ of soils. ASTM, 6

Babula, P., Adam, V., Havel, L., y Kisek, R. (2012). Cadmium Accumulation by Plants of Brassicaceae Family and Its Connection with Their Primary and Secondary Metabolism. En N. Anjun, I. Ahmad, E. Pereira, A. Duarte, S. Umar, y N. Khan, The Plant Family Brassicaceae. Contribution Towards Phytoremediation (págs. 71-98). Moscú: Editorial Board

Chamizo, A., Ferrera, R., Gonzáles, M., y Ortiz, C. (2009). Inoculación de alfalfa con hongos micorrízicos arbusculares $\mathrm{y}$ rizobacterias en dos tipos de suelo. TerraLatinoamericana Volumen $27, \mathrm{~N}^{\circ} 3$, 9

Chinmayee, D., Mahesh, B., Pradesh, S., y Mini, I. (2012). The Assessment of Phytoremediation Potential of Invasive Weed Amaranthus Spinosus L. Appl. Biochem Biotechnol, 10

Cruz, M., y Guzmán, A. (2007). La contaminación de suelos y aguas. Su prevención con nuevas sustancias natruales. España: Universidad de Sevilla

Datta, R., Geiger, E., y Sarkar, D. (2013). Lead an Phytoremediation. Michinga: Encyclopedia of Metalloproteins

DeSouza, E., Guimaraes, L., Chaves, E., Ribeiro, B., DosSantos, E., DaCosta, E., y otros. (2012). Assessing the Tolerance of Castor Bean to $\mathrm{Cd}$ and $\mathrm{Pb}$ for Phytoremediation Purposes. Biol Trace Elem Res, 8

Diez, J., Kidd, P., y Monterroso, C. (2009). Biodisponibilidad de metales en suelos y acumulación en plantas en el área de Trás-os-Montes (NE Portugal): influencia del material original. España: Sociedad española de la Ciencia del suelo, 17

EPA. (2005). EPA 3010a. Acid digestion of aqueous samples and extracts for total metals for analysis by FLAA or ICP Spectroscopy. Standard methods ed-212005, 5

Flores, A. (2010). Influencia en seis híbridos de girasol (Helianthus annus), con aplicación de boro, su comportamiento agronómico y el rendimiento, en la granja experimental E.C.A.A. Quito: Pontificia Universidad Católica del Ecuador Sede Ibarra 
Guanopatín, M. (2012). Aplicación de Biol en el cultivo establecido de Alfalfa (Medicago sativa). Universidad Técnica de Ambato

Gupta, D., Huang, H., y Corpas, F. (2013). Lead tolerance in plantas: strategies for phytoremediation. Environ Sci Pollut Res, 12

Herrera, K. (2009). Evaluación de la contaminación por plomo en suelos del cantón Sitio del Niño Municipio de San Juan Opico departamento de la Libertad. Tesis Licenciatura, Universidad de El Salvador. Universidad de El Salvador, 129

Jácome, E. (2011). Perforaciones y estudios del suelo. Unidad de Metro Quito, 2

Lee, J. (2013). An Overview of Phytoremediation as a Potentially Promising Technology for Environmental Pollution Control. Biotechnology and Bioprocess Engineering, 9

López, E., y Torija, E. (2006). Caracterización y tipificación de mostazas comerciales. Universidad Complutense de Madrid, 304

Manasi, Rajesh, V., y Rajesh, N. (2014). Adsorption isotherms, kinetics and thermodynamic studies towards understanding the interaction between a microbe inmobilized polysaccharide maatrix and lead. Chemical Engineering Journal, 10

NOM. (2002). Norma Oficial Mexicana NOM199-SSA1-2000, Salud ambiental. Niveles de plomo en sangre y acciones como criterios para proteger la salud de la población expuesta no ocupacionalmente. Norma Oficial Mexicana, 20

Nouri, J., Lorestani, B., Yousefi, N., y Khorasani, N. (2011). Phytoremediation potential of native plants grown in the vicinity of Ahangaran lead-zinc mine (Hamedan, Iran). Environ Earth Sci. , 6

Pacheco, M., Pimentel, J., y Roque, W. (2010). Cinética de la bioadsorción de iones cadmio (II) y plomo (II) de soluciones acuosas por biomasa residual de café (Coffea arabica L.). Revista de la Sociedad Química del Perú V.76 N.3, 10.

Peng, X., Yang, B., y Deng, D. (2012). Lead tolerance and accumulation in three cultivars of Eucalyptus urophylla XE.grandis: implication for phytoremediation. Environ Earth Sci., 6

Peralta. (2009). Amaranto y Ataco preguntas y respuestas. Ecuador: Programa Nacional de Leguminosas y Granos Andinos INIAP. Vol. 4

Peralta, E. (2012). El amaranto en Ecuador "Estado del Arte". PRONALEG-GA, INIAP, 43

Puga, S., Sosa, M., Lebgue, T., Quintana, C., y Campos, A. (2006). Contaminación por metales pesados en el suelo provocado por la industria minera. Ecología aplicada, 149-155

RECNAT. (2002). Norma Oficial Mexicana NOM-021-SEMARNAT-2000 que establece las especificaciones de fertilidad, salinidad y clasificación de suelos, estudio, muestreo y análisis. Secretaría de Medio Ambiente y Recursos Naturales, 85

Redín, L. (2009). Caracterización física, química y nutricional de dos ecotipos de acelga (beta vulgaris L.) cultivados en el Ecuador como un aporte a la actualización de la norma INEN N 1749 "Hortalizas frescas, acelga requisitos". Universidad Tecnológica Equinoccial, 198

Salas, M., y Boradonenko, A. (2009). Insectos asociados al amaranto amaranthus hupocondriacus L. (amaranthaceae) en Irapuato, Guanajuato, México. Universidad de Guanajuato, 7

Sarvajeet, G., Naser, A., Iqbal, A., Thangavel, P., Sridevi, G., Pacheco, A., y otros. (2012). Metal Hyperaccumulation and Tolerance in Alyssum, Arabidopsis and Thlaspi: An Overview. En N. Anjum, I. Ahmad, E. Pereira, A. Duarte, S. Umar, y N. Khan, The Plant Family Brassicaceae. Contribution Towards Phytoremediation (págs. 99137). Moscú: Board

Seth, C., Misra, V., y Singh, R. (2011). EDTAenhanced lead phytoremediation in sonflower (Helianthus annuus L.) hydroponic culture. Plant Soil, 12 
Singh, A., y Fulekar, M. (2012). Phytoremediation of Heavy Metals by Brassica juncea in Aquatic and Terrestrial Environment. En N. Anjum, I. Ahmad, E. Pereira, A. Duarte

Suthar, V., Memon, K., y Hassa, M. (2014). EDTA- enhanced phytoremediation of contaminated calcareous soils: heavy metal bioavailability, extractability, and uptake by maize and sesbania. Environ Monit Assess, 12

Volke, T., Velasco, J., y Pérez, D. (2005). Suelos contaminados por metales y metaloides: Muestreo y alternativas para su remediación. Instituto Nacional de ecología: Secretaría de medio ambiente y recursos naturales 